\title{
Electroweak phase transition beyond the Standard Model
}

\section{Dolgopolov}

Samara State University

E-mail: dolg@ssu.samara.ru

\section{Dubinin*}

Skobeltsyn Institute of Nuclear Physics, Moscow State University

E-mail: dubininetheory.sinp.msu.ru

\section{E. Rykova}

Samara State University

E-mail: elza@rambler.ru

In the framework of the two-Higgs-doublet model with temperature-dependent effective parameters $\lambda_{1, \ldots 7}(T)$ we define the four bifurcation sets which appear as a consequence of the condition $\operatorname{det} \partial^{2} U_{i j} / \partial v_{i} \partial v_{j}=0$ for the stability matrix. Numerical investigations of the electroweak phase transition are performed for the full MSSM parameter space $\left(m_{H^{ \pm}}, \operatorname{tg} \beta, A_{t, b}, \mu, m_{Q}, m_{U}, m_{D}\right)$ using the temperature-dependent effective parameters $\lambda_{1}-\lambda_{5}(T)$ calculated at the one-loop in the imaginary time formalism. Four types of transitions can take place in the MSSM background field space $\left(v_{1}, v_{2}\right)$. They are different in the direction, defined by a critical angle in the $\left(v_{1}, v_{2}\right)$ plane, and in the strength, which is estimated by means of Shaposhnikov criteria $v_{c} / T_{c}>1$. Extensive regions in the $\left(A_{t, b}, \mu\right)$ plane correspond to the strongly first order phase transition. The case of light top quark superpartner is favored, giving an acceptable configurations of surfaces for extrema of the effective potential.

The XIXth International Workshop on High Energy Physics and Quantum Field Theory

8-15 September 2010

Golitsyno, Moscow, Russia

\footnotetext{
*Speaker.
} 


\section{Introduction}

Theories of cosmological evolution usually include an assumption that rather hot early universe with the temperature of the order of a few hundreds of $\mathrm{GeV}$ and unbroken $S U(2)_{w} \times U(1)_{Y}$ symmetry of the ground state (characterized by zero Higgs field vacuum expectation value - v.e.v.) was transformed with the decrease of temperature to the state with nonzero Higgs field v.e.v., so as a result of such electroweak phase transition the electroweak symmetry was broken spontaneously down to $U(1)_{e m}$. Investigation of the temperature evolution beyond the Standard Model is important as a basis for models which describe generation of the baryon asymmetry, nature of the dark matter and also for some inflationary models of early universe.

In the following we are going to analyze the equilibrium states of the effective finite-temperature potential (free energy) for the two-Higgs doublet model (THDM)

$$
\begin{aligned}
& U_{e f f}\left(\Phi_{1}, \Phi_{2}\right)=-\mu_{1}^{2}\left(\Phi_{1}^{\dagger} \Phi_{1}\right)-\mu_{2}^{2}\left(\Phi_{2}^{\dagger} \Phi_{2}\right)-\mu_{12}^{2}\left(\Phi_{1}^{\dagger} \Phi_{2}\right)-\mu_{12}^{2}\left(\Phi_{2}^{\dagger} \Phi_{1}\right)+\lambda_{1}(T)\left(\Phi_{1}^{\dagger} \Phi_{1}\right)^{2}+\lambda_{2}(T)\left(\Phi_{2}^{\dagger} \Phi_{2}\right)^{2} \\
& +\lambda_{3}(T)\left(\Phi_{1}^{\dagger} \Phi_{1}\right)\left(\Phi_{2}^{\dagger} \Phi_{2}\right)+\lambda_{4}(T)\left(\Phi_{1}^{\dagger} \Phi_{2}\right)\left(\Phi_{2}^{\dagger} \Phi_{1}\right)+\frac{\lambda_{5}(T)}{2}\left(\Phi_{1}^{\dagger} \Phi_{2}\right)\left(\Phi_{1}^{\dagger} \Phi_{2}\right)+\frac{\lambda_{5}^{*}(T)}{2}\left(\Phi_{2}^{\dagger} \Phi_{1}\right)\left(\Phi_{2}^{\dagger} \Phi_{1}\right)+ \\
& +\lambda_{6}(T)\left(\Phi_{1}^{\dagger} \Phi_{1}\right)\left(\Phi_{1}^{\dagger} \Phi_{2}\right)+\lambda_{6}^{*}(T)\left(\Phi_{1}^{\dagger} \Phi_{1}\right)\left(\Phi_{2}^{\dagger} \Phi_{1}\right)+\lambda_{7}(T)\left(\Phi_{2}^{\dagger} \Phi_{2}\right)\left(\Phi_{1}^{\dagger} \Phi_{2}\right)+\lambda_{7}^{*}(T)\left(\Phi_{2}^{\dagger} \Phi_{2}\right)\left(\Phi_{2}^{\dagger} \Phi_{1}\right)
\end{aligned}
$$

where the temperature-dependent background field v.e.v.'s are $\left\langle\Phi_{1}\right\rangle=\left(0, v_{1}(T)\right) / \sqrt{2}$ and $\left\langle\Phi_{2}\right\rangle=$ $\left(0, v_{2}(T)\right) / \sqrt{2}$. Local properties of $U_{\text {eff }}\left(v_{1}, v_{2}, \lambda_{1}, \ldots \lambda_{7}\right)$ are defined by a number of well-known theorems in the framework of the catastrophe theory (Morse and Thom theorems for the reduction of a potential function to the canonical form by a nonlinear transformation [1]). They describe properties of the stationary state $\nabla U_{e f f}\left(v_{1}, v_{2}\right)=0$ defined by the stability matrix (also called the Hessian) $U_{i j}=\partial^{2} U_{e f f} / \partial v_{i} \partial v_{j}$.

\section{Bifurcation sets in the two-Higgs-doublet model}

Thermodynamical evolution of the two-Higgs-doublet potential (1.1) as a function of the two variables of state $v_{1}$ and $v_{2}$ and six temperature-dependent control parameters $\lambda_{1}(T), \ldots \lambda_{7}(T)$

$$
U\left(v_{1}, v_{2}\right)=-\frac{\mu_{1}^{2}}{2} v_{1}^{2}-\frac{\mu_{2}^{2}}{2} v_{2}^{2}-\mu_{12}^{2} v_{1} v_{2}+\frac{\lambda_{1}}{4} v_{1}^{4}+\frac{\lambda_{2}}{4} v_{2}^{4}+\frac{\lambda_{345}}{4} v_{1}^{2} v_{2}^{2}+\frac{\lambda_{6}}{2} v_{1}^{3} v_{2}+\frac{\lambda_{7}}{2} v_{1} v_{2}^{3}
$$

from some high temperature $T$ of the order of several hundred $\mathrm{GeV}$ down to zero is defined not only by a temperature evolution of effective parameters $\lambda_{1, \ldots 7}(T)$ but also by the parametric dependence of $\lambda_{1, \ldots 7}$ on masses, couplings and mixing angles which exist in different sectors of a model under consideration. In the following numerical analysis we shall use the one-loop temperaturedependent $\lambda_{1, \ldots 7}$ calculated symbolically in the framework of the MSSM, see [2,3]. We denote $\lambda_{345}=\lambda_{3}+\lambda_{4}+\operatorname{Re} \lambda_{5}$. Conditions of the extremum $\nabla U\left(v_{1}, v_{2}\right)=0$ distinguishing an isolated (or nondegenerate) critical points

$$
\begin{aligned}
& \mu_{1}^{2}=\lambda_{1} v_{1}^{2}+\lambda_{345} \frac{v_{2}^{2}}{2}-\operatorname{Re} \mu_{12}^{2} \operatorname{tg} \beta+\frac{v^{2} s_{\beta}^{2}}{2}\left(3 \operatorname{Re} \lambda_{6} \operatorname{ctg} \beta+\operatorname{Re} \lambda_{7} \operatorname{tg} \beta\right), \\
& \mu_{2}^{2}=\lambda_{2} v_{2}^{2}+\lambda_{345} \frac{v_{1}^{2}}{2}-\operatorname{Re} \mu_{12}^{2} \operatorname{ctg} \beta+\frac{v^{2} c_{\beta}^{2}}{2}\left(\operatorname{Re} \lambda_{6} \operatorname{ctg} \beta+3 \operatorname{Re} \lambda_{7} \operatorname{tg} \beta\right),
\end{aligned}
$$


where

$$
\operatorname{Re} \mu_{12}^{2}=\sin \beta \cos \beta\left[m_{A}^{2}+\frac{v^{2}}{2}\left(2 \operatorname{Re} \lambda_{5}+\operatorname{Re} \lambda_{6} \operatorname{ctg} \beta+\operatorname{Re} \lambda_{7} \operatorname{tg} \beta\right)\right],
$$

are also mentioned as the minimization conditions which set to zero the linear terms in the physical Higgs fields $h, H$ and $A$ and ensure a local extremum at any point of the surface $U_{e f f}\left(v_{1}, v_{2}\right)$ in the background field space (see e.g. [4]) ${ }^{1}$ Important input parameters of the two-doublet potential are $\operatorname{tg} \beta=v_{2} / v_{1}$ and the charged Higgs boson mass

$$
m_{H^{ \pm}}^{2}=m_{W}^{2}+m_{A}^{2}-\frac{v^{2}}{2}\left(\operatorname{Re} \Delta \lambda_{5}-\Delta \lambda_{4}\right)
$$

where the effective temperature-dependent mass of the longitudinal $W$-boson is $m_{W_{L}}^{2}(v, T)=m_{W}^{2}(v)+$ $\Pi_{W_{L}}(T), \Pi_{W_{L}}(T)=5 g_{2}^{2} T^{2} / 2$ (with the one-loop Standard Model and third-generation squarks contributions included in the polarization operator; $m_{W}^{2}=v^{2} g_{2}^{2} / 2$ ). Thermal evolution of the system in the $v_{1}(T), v_{2}(T)$ space is, as a rule, an equilibrium process, which conserves the minimization of $U$ with respect to the scalar fields oscillation in the extremum defined by current values of $v_{1}(T), v_{2}(T)$. Such assumption continuously admits the interpretation of the system in terms of scalar mass eigenstates $h, H$ and $A$, then $\mu_{1}^{2}, \mu_{2}^{2}$ and $\mu_{12}^{4}$ can be expressed by means of the effective parameters $\lambda_{1, \ldots .7}[4]$.

For simplicity we consider the case $\lambda_{6}=\lambda_{7}=0$. The two-doublet Higgs potential without $\lambda_{6}$ and $\lambda_{7}$ terms has been considered in the context of discrete Peccei-Quinn symmetry [5]. Nonisolated (or degenerate) critical points in the $v_{1}, v_{2}$ plane are defined by the condition $\operatorname{det} \partial^{2} U / \partial v_{i} \partial v_{j}=0$ which can be written in the form

$$
\operatorname{det}\left\|\begin{array}{cc}
2 \lambda_{1} v_{1}^{2}+\mu_{12}^{2} \frac{v_{2}}{v_{1}} & -\mu_{12}^{2}+\lambda_{345} v_{1} v_{2} \\
-\mu_{12}^{2}+\lambda_{345} v_{1} v_{2} & 2 \lambda_{2} v_{2}^{2}+\mu_{12}^{2} \frac{v_{1}}{v_{2}}
\end{array}\right\|=0
$$

where the minimization conditions (2.2) and (2.3) (or, equivalently, the conditions for isolated points of $\left.U\left(v_{1}, v_{2}\right)\right)$ have been substituted. The system of two nonlinear equations for $v_{1}, v_{2}$

$$
\begin{aligned}
& \lambda_{1} v_{1}^{3}+\frac{\lambda_{345}}{2} v_{1} v_{2}^{2}-\mu_{1}^{2} v_{1}-\mu_{12}^{2} v_{2}=0 \\
& \lambda_{2} v_{2}^{3}+\frac{\lambda_{345}}{2} v_{1}^{2} v_{2}-\mu_{2}^{2} v_{2}-\mu_{12}^{2} v_{1}=0
\end{aligned}
$$

can be factorized by the rotation in the $v_{1}, v_{2}$ plane

$$
v_{1}=\bar{v}_{1} \cos \bar{\beta}-\bar{v}_{2} \sin \bar{\beta}, \quad v_{2}=\bar{v}_{1} \sin \bar{\beta}+\bar{v}_{2} \cos \bar{\beta}
$$

where

$$
\sin ^{2} \bar{\beta}=\frac{1}{2} \pm \frac{\left|\mu_{1}^{2}-\mu_{2}^{2}\right|}{2 \sqrt{\left(\mu_{1}^{2}-\mu_{2}^{2}\right)^{2}+4 \mu_{12}^{4}}}, \quad \cos ^{2} \bar{\beta}=\frac{1}{2} \mp \frac{\left|\mu_{1}^{2}-\mu_{2}^{2}\right|}{2 \sqrt{\left(\mu_{1}^{2}-\mu_{2}^{2}\right)^{2}+4 \mu_{12}^{4}}}
$$

\footnotetext{
${ }^{1}$ Although only the CP-conserving limit is considered, we keep the notation of real parts for the variables where a phase factor could appear in the general case.
} 
Then the factorized equations (2.6) are

$$
\begin{aligned}
& \bar{v}_{1}\left(\lambda_{1} \bar{v}_{1}^{2}+\frac{\lambda_{345}}{2} \bar{v}_{2}^{2}-\bar{\mu}_{1}^{2}\right)=0 \\
& \bar{v}_{2}\left(\lambda_{2} \bar{v}_{2}^{2}+\frac{\lambda_{345}}{2} \bar{v}_{1}^{2}-\bar{\mu}_{2}^{2}\right)=0
\end{aligned}
$$

where

$$
\bar{\mu}_{1,2}^{2}=\frac{1}{2}\left(\mu_{1}^{2}+\mu_{2}^{2} \pm \sqrt{\left(\mu_{1}^{2}-\mu_{2}^{2}\right)^{2}+4 \mu_{12}^{4}}\right)
$$

and the four types of bifurcation sets defined by the stability matrices $U_{i j}\left(v_{1}, v_{2}\right)$ can be easily found

(1) $\lambda_{1} \bar{v}_{1}^{2}+\frac{\lambda_{345}}{2} \bar{v}_{2}^{2}-\bar{\mu}_{1}^{2}=0$ and $\lambda_{2} \bar{v}_{2}^{2}+\frac{\lambda_{345}}{2} \bar{v}_{1}^{2}-\bar{\mu}_{2}^{2}=0, U_{i j}\left(v_{1}, v_{2}\right)=\left\|\begin{array}{cc}2 \lambda_{1} \bar{v}_{1}^{2} & \lambda_{345} \bar{v}_{1} \bar{v}_{2} \\ \lambda_{345} \bar{v}_{1} \bar{v}_{2} & 2 \lambda_{2} \bar{v}_{2}^{2}\end{array}\right\|$

(2) $\lambda_{1} \bar{v}_{1}^{2}-\bar{\mu}_{1}^{2}=0$ and $\bar{v}_{2}=0, U_{i j}\left(v_{1}, v_{2}\right)=\left\|\begin{array}{cc}2 \lambda_{1} \bar{v}_{1}^{2} & 0 \\ 0 & -\bar{\mu}_{2}^{2}+\frac{\lambda_{345}}{2} \bar{v}_{1}^{2}\end{array}\right\|$

(3) $\bar{v}_{1}=0$ and $\lambda_{2} \bar{v}_{2}^{2}-\bar{\mu}_{2}^{2}=0, U_{i j}\left(v_{1}, v_{2}\right)=\left\|\begin{array}{cc}-\mu_{1}^{2}+\frac{\lambda_{345}}{2} \bar{v}_{2}^{2} & 0 \\ 0 & 2 \lambda_{2} \bar{v}_{2}^{2}\end{array}\right\|$

(4) $\bar{v}_{1}=0$ and $\bar{v}_{2}=0, U_{i j}\left(v_{1}, v_{2}\right)=-\left\|\begin{array}{cc}\bar{\mu}_{1}^{2} & 0 \\ 0 & \bar{\mu}_{2}^{2}\end{array}\right\|$
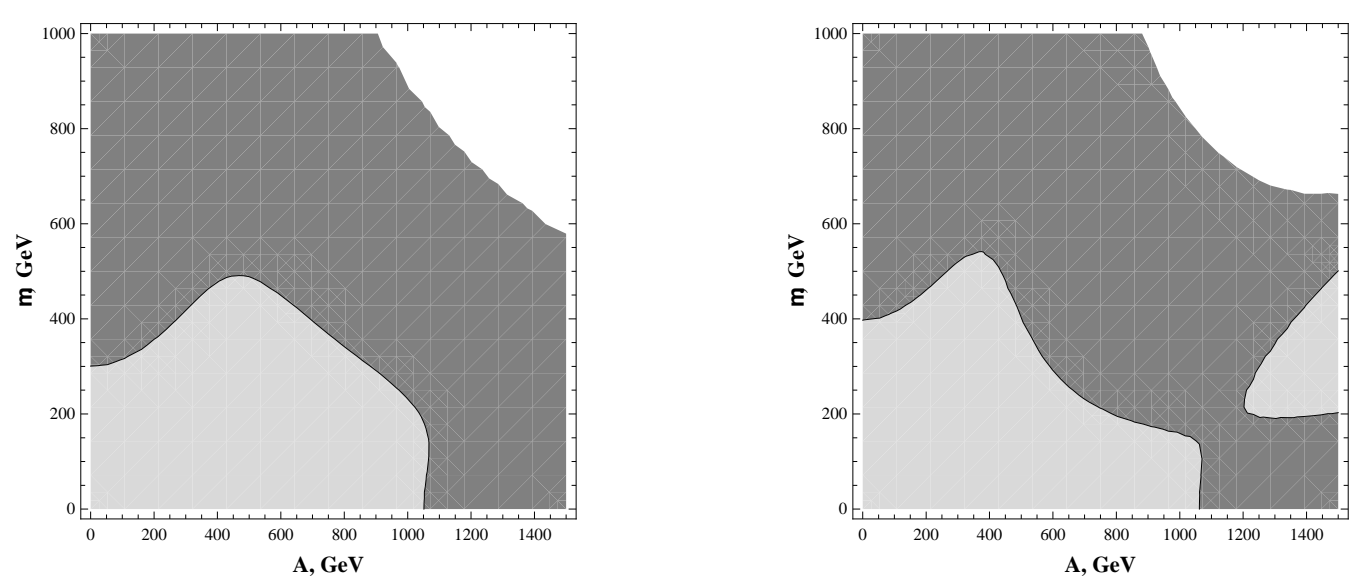

Figure 1: Left panel: bifurcation set (1), contour of negatively defined determinant $\lambda_{1} \lambda_{2}-\lambda_{345}^{2} / 4$ (dark grey area), see Eq.(2.11), in the $\left(A_{t}=A_{b}, \mu\right)$ plane at the temperature $150 \mathrm{GeV}$. The squark sector parameter values $m_{Q}=500 \mathrm{GeV}, m_{U}=200 \mathrm{GeV}, m_{D}=800 \mathrm{GeV}$ are in line with the case of light stop quark. Right panel: bifurcation set (2), contour of negatively defined determinant $\left(4 \lambda_{1}+\lambda_{345}\right)\left(4 \lambda_{2}+\lambda_{345}\right)-\left(3 \lambda_{345}-\lambda_{1}-\lambda_{2}\right)^{2}$ (dark grey area), see Eq.(2.17), at the same parameter values.

Bifurcation set of the case (1), which is defined by $\operatorname{det} \partial^{2} U / \partial v_{i} \partial v_{j}=0$, can be understood in the elementary language. The surface of stationary points $U_{\text {eff }}\left(v_{1}, v_{2}\right)=-\left(\lambda_{1} v_{1}^{4}+\lambda_{2} v_{2}^{4}+\right.$ $\left.\lambda_{345} v_{1}^{2} v_{2}^{2}\right) / 4$ is positively defined and unbounded from above if the Sylvester's criteria for the 
quadratic form $U_{\text {eff }}\left(v_{1}^{2}, v_{2}^{2}\right)$ is respected

$$
\lambda_{1}<0, \quad \lambda_{2}<0, \quad \lambda_{1} \lambda_{2}-\frac{\lambda_{345}^{2}}{4}<0
$$

At the critical temperature defined by the equation $\lambda_{1} \lambda_{2}-\lambda_{345}^{2} / 4=0$ the positively defined potential surface of stationary points starts to develop the saddle configuration which is unbounded from below. The "flat direction" at the critical temperature which is developed at the angle $\operatorname{tg} 2 \theta=$ $2 \lambda_{345} /\left(\lambda_{1}^{2}-\lambda_{2}^{2}\right)$, or

$$
\operatorname{tg} \theta=\left|\frac{\lambda_{345}}{\left|\lambda_{1}-\lambda_{2}\right|-\sqrt{\left(\lambda_{1}-\lambda_{2}\right)^{2}+\lambda_{345}^{2}}}\right|
$$

is defined by the control parameters $\lambda_{1}(T), \lambda_{2}(T)$ and $\lambda_{345}(T)$ not depending on the $v_{1}$ and $v_{2}$. The contour for Sylvester's criteria (2.11) is shown in Fig. 1 at the temperature $T=150 \mathrm{GeV}$ in the $\left(A=A_{t}=A_{b}, \mu\right)$ plane. The squark mass parameters $m_{Q}, m_{U}$ and $m_{D}$ are fixed, the $(A, \mu)$ parameters are chosen in the vicinity of the contours which separate positively and negatively defined $\lambda$-parameters in (2.11). The critical temperature in this case is slightly above $120 \mathrm{GeV}$, insignificantly dependent on the values of $\left(A_{t, b}, \mu\right)$ if they are changing along the contours in Fig.1, separating the light grey and the dark grey areas. The strength of the electroweak phase transition
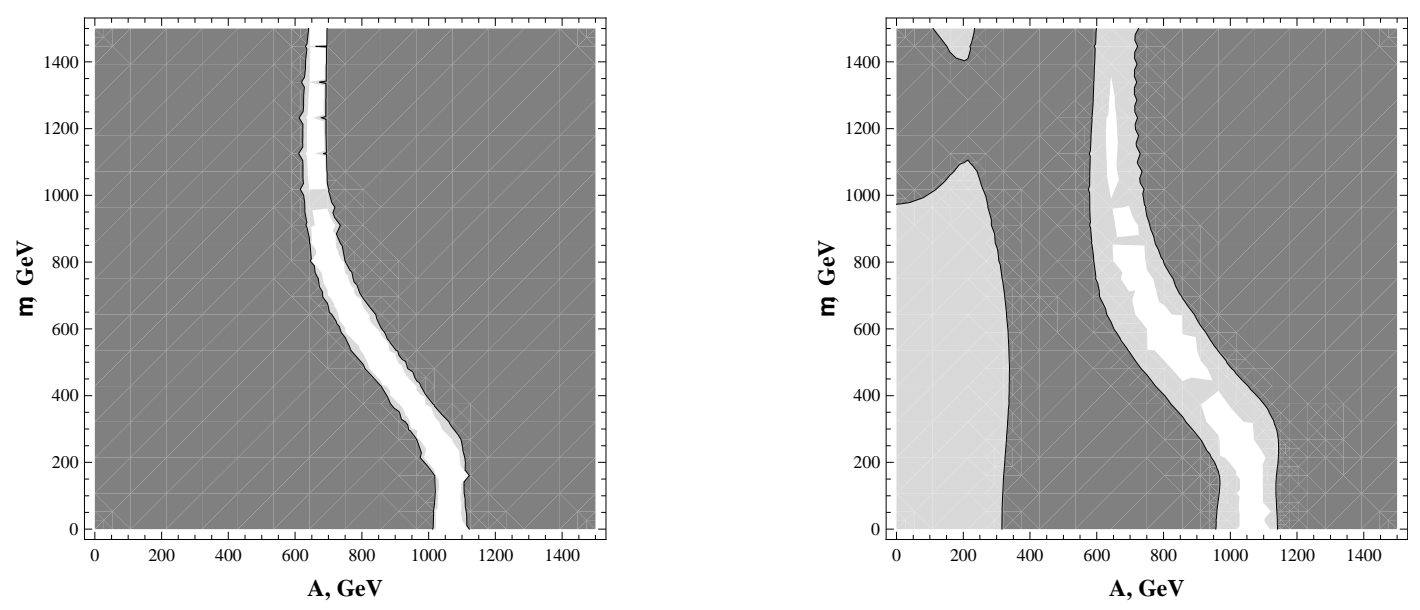

Figure 2: Contours for the criteria $\frac{v_{c}}{T_{c}}=1$ in the $\left(A_{t}=A_{b}, \mu\right)$ plane. In the light grey regions $\frac{v_{c}}{T_{c}}>1$. In order to include qualitatively the effect of $E_{M S S M}$, for the left plot $E=2 E_{S M}$ and for the right plot $E=4 E_{S M}$. $\lambda_{6}=\lambda_{7}=0$, charged Higgs boson mass $m_{H^{ \pm}}=150 \mathrm{GeV}$. Set (A), the case of light stop, is used for the squark sector parameter values $\left(m_{Q}=500 \mathrm{GeV}, m_{U}=200 \mathrm{GeV}, m_{D}=800 \mathrm{GeV}\right)$.

along the direction (2.12) can be roughly estimated using the equation

$$
\frac{v\left(T_{c}\right)}{T_{c}}=\frac{2 \sqrt{2} E}{\lambda(\theta)}
$$

where $E$ is a temperature-independent factor in front of the cubic term $-E T v^{3}$ in the effective potential rewritten in the polar coordinates $\left(v=\sqrt{v_{1}^{2}+v_{2}^{2}}, \theta=\arctan \left(v_{2} / v_{1}\right)\right)$, and $\lambda(\theta)$ is a factor in front of the quartic term $v^{4} / 4$. The cubic term is given by corrections coming from the 
resummation of the multiloop diagrams in the infrared region. In the case of a heavy stop which decouples [6], the effective potential is similar to Standard Model potential and

$$
E_{S M}=\frac{2 \sqrt{2}}{48 \pi}\left[2 g_{2}^{3}+\left(g_{1}^{2}+g_{2}^{2}\right)^{3 / 2}\right]=\frac{\sqrt{2}}{3} \frac{\left(2 m_{W}^{2}+m_{Z}^{3}\right)}{\pi v^{3}} .
$$

In the case of a light stop one can use an approximation $E=E_{S M}+E_{M S S M}$, where an additional term [7]

$$
E_{M S S M}=\frac{2 \sqrt{2}}{3 \pi v^{3}} m_{t}^{3}\left(1-\frac{\tilde{A}_{t}^{2}}{m_{Q}^{2}}\right)^{\frac{3}{2}}
$$

stop mixing parameter here $\tilde{A}_{t}=A_{t}-\mu / \operatorname{tg} \beta$. The quartic term along the direction (2.12) can be written in the form

$$
\lambda(\theta)=-\frac{\lambda_{1}+\lambda_{345} \operatorname{tg}^{2} \theta+\lambda_{2} \operatorname{tg}^{4} \theta+2 \lambda_{6} \operatorname{tg} \theta+2 \lambda_{7} \operatorname{tg}^{3} \theta}{\left(1+\operatorname{tg}^{2} \theta\right)^{2}} .
$$

The condition $v_{c} / T_{c}>1$ [8], necessary to avoid sphaleron transitions which erase the baryon asymmetry initially generated at the electroweak phase transition, can be respected in a rather extensive regions of the $(A, \mu)$ plane. The contours of $v_{c} / T_{c}>1$ in the $(A, \mu)$ plane (see Fig.2) separate the regions not only around the origin $(A, \mu)=(0,0)$, but also the areas with $(A, \mu)$ of the order of $1 \mathrm{TeV}$, where the quartic term $\lambda(\theta)$ changes sign crossing zero along the flat direction (2.12).

For the general case of nonzero $\lambda_{6}$ and $\lambda_{7}$ which are defined at the one-loop in the finitetemperature MSSM, see [2,3], the effective potential $U_{\text {eff }}\left(v_{1}, v_{2}\right)=-\left(\lambda_{1} v_{1}^{4}+\lambda_{2} v_{2}^{4}+\lambda_{345} v_{1}^{2} v_{2}^{2}+\right.$ $\left.2 \lambda_{6} v_{1}^{3} v_{2}+2 \lambda_{7} v_{1} v_{2}^{3}\right) / 4$ always demonstrates a saddle configuration for the surface of stationary points, which slopes become steeper with an increase of the temperature.

Bifurcation sets in the cases (2) and (3) are different from the bifurcation set in the case (1). For the case (2) we found

$$
\left(4 \lambda_{1}+\lambda_{345}\right) v_{1}^{4}+\left(4 \lambda_{2}+\lambda_{345}\right) v_{2}^{4}+\left(6 \lambda_{345}-2 \lambda_{1}-2 \lambda_{2}\right) v_{1}^{2} v_{2}^{2}=0
$$

The regions of positively and negatively defined $\lambda_{1}$ and $\lambda_{2}$ and the contour for Sylvester's criteria for the form (2.17) are shown in Fig.1 at the temperature $T=150 \mathrm{GeV}$ in the $\left(A=A_{t}=A_{b}, \mu\right)$ plane. The phase transition for the case (2) is developed in the direction $\theta$ of the $\left(v_{1}, v_{2}\right)$ plane

$$
\operatorname{tg} 2 \theta=\frac{4\left(3 \lambda_{345}-\lambda_{1}-\lambda_{2}\right)}{\left(4 \lambda_{1}+\lambda_{345}\right)^{2}-\left(4 \lambda_{2}+\lambda_{345}\right)^{2}}
$$

Bifurcation set in the case (4) $\bar{v}_{1}=0$ and $\bar{v}_{2}=0$ defined by the equation $\bar{\mu}_{1}^{2} \bar{\mu}_{2}^{2}=0$ can also be understood on the elementary level as a result of the diagonalization of the effective potential $U_{\text {eff }}=-\frac{\mu_{1}^{2}}{2} v_{1}^{2}-\frac{\mu_{2}^{2}}{2} v_{2}^{2}-\mu_{12}^{2} v_{1}^{2} v_{2}^{2}$ by the rotation (2.7), giving the form $U_{\text {eff }}=-\bar{\mu}_{1}^{2} \bar{v}_{1}^{2}-\bar{\mu}_{2}^{2} \bar{v}_{2}^{2}$. In such regime all other sectors of the model decouple, dominant contribution is given by the "mass term" of dimension two in the fields. The critical temperature is defined by the equation [10]

$$
\mu_{1}^{2} \mu_{2}^{2}=\mu_{12}^{4}
$$

which is equivalent to $\bar{\mu}_{1}^{2}=0$ or $\bar{\mu}_{2}^{2}=0$. If $\lambda_{i}$ are small enough the critical angle can be expressed as

$$
\operatorname{tg} 2 \theta=\operatorname{tg} 2 \beta \frac{1}{\left(\frac{v^{2}}{2 m_{A}^{2}}-\alpha_{1}\right)} \frac{1}{\frac{2 \lambda_{1} \cos ^{2} \beta-2 \lambda_{2} \sin ^{2} \beta}{\cos 2 \beta}-\lambda_{345}+\frac{2 m_{A}^{2}}{v^{2}}+\alpha_{2}}
$$


where

$$
\alpha_{1}=\frac{\lambda_{5}}{2}+\frac{1}{4}\left(\lambda_{6} \operatorname{ctg} \beta+\lambda_{7} \operatorname{tg} \beta\right), \quad \alpha_{2}=\lambda_{6}(\operatorname{tg} 2 \beta-\operatorname{ctg} \beta)-\lambda_{7}(\operatorname{tg} \beta+\operatorname{tg} 2 \beta)
$$

A number of contours in the MSSM parameter space for this case can be found in [3].

\section{Summary}

Four types of bifurcation sets for the effective finite-temperature two-Higgs-doublet potential $U_{\text {eff }}\left(v_{1}, v_{2}\right)$ are found. In the MSSM the bifurcation set (1) defined by Eq.(2.11) develops a phase transition in the direction fixed by Eq.(2.12) in the $\left(v_{1}, v_{2}\right)$ plane. Numerical investigations in the MSSM show that the parameter set with soft SUSY breaking parameters $A$ and $\mu$ of the order of $1 \mathrm{TeV}$, inherent to known MSSM scenarios [9] of strong CP violation (CPX), combined with the squark-Higgs boson sector parameters $m_{Q}=500 \mathrm{GeV}, m_{U}=200 \mathrm{GeV}$ and $m_{D}=800 \mathrm{GeV}$, gives an acceptable configuration of the surface for stationary points (paraboloid with a global minimum at the origin at high temperatures and a saddle at low temperatures). In the region of the MSSM parameter space characterized by $m_{Q}, m_{U}$ and $m_{D}$ as indicated above or close to these values, the top scalar quark is light (not much above $100 \mathrm{GeV}$ ). The bifurcation contour (also called the separatrix in the catastrophe theory terminology) in the $(A, \mu)$ plane for the set (1) is shown in Fig.1, left. Alternatively, parameter sets with the light sbottom and heavy stop always, both at a high and at a low temperatures, give a saddle configuration for the surface of stationary points. The bifurcation sets (2) and (3) are similar, demonstrating a phase transition in the direction defined by Eq.(2.18) in the $\left(v_{1}, v_{2}\right)$ plane. The bifurcation contours in the $(A, \mu)$ plane for the set (2) are shown in Fig.1, right. Again, only the parameter set with the light stop demonstrates a necessary configuration of the equilibrium surfaces. The bifurcation set (4) includes a phase transition in the direction of Eq.(2.20) at the temperature defined by Eq.(2.19). Analogous case was analyzed earlier in the literature in the context of the one-dimensional effective potential. Summarizing, in all four cases the global minimum at the origin $v_{1}=v_{2}=0, U_{\text {eff }}(0,0)=0$ at high temperatures is transformed to a local minimum with $U_{e f f}\left(v_{1}, v_{2}\right)<0$ at a lower temperature for the MSSM parameter sets with a light superpartner of the top quark, but the directions of transition to this minimum in the $\left(v_{1}, v_{2}\right)$ plane are different.

Rough estimate for the strength of the first order phase transition using the approximation for $v_{c} / T_{c}$ defined by Eq.(2.13), when the mimimum at the origin is on the same level with the minimum at $v_{c}$, separated by a low potential barrier along a critical direction, demonstrates a rather extensive regions of strong first order phase transition in the $(A, \mu)$ plane (Fig.2). They increase with the decrease of the quartic term parameter $\lambda(\theta)$, Eq.(2.16), being less significantly dependent on the factor $E$, see Eq.(2.13), in front of the cubic term.

Acknowlegements. Work was partially supported by grants ADTP 3341, RFBR 10-02-00525-a, NS 1456.2008.2 and FAP contract 5163. 


\section{References}

[1] R. Gilmore, Catastrophe theory for scientists and engineers, John Wiley \& Sons, New York-Chichester-Brisbane-Toronto, 1981

[2] A.Borisov, M.Dolgopolov, M.Dubinin, E.Rykova, Analytic expressions for threshold corrections to the finite-temperature Higgs potential in the minimal supersymmetric standard model, Phys.Atom.Nucl., 2009, 72, P.167 (Yad.Fiz., 2009, 72, P.175).

[3] M.Dolgopolov, M.Dubinin, E.Rykova, Threshold corrections to the MSSM finite-temperature Higgs potential, arXiv:0901.0524 [hep-ph]

[4] E.N. Akhmetzyanova, M.V. Dolgopolov, M.N. Dubinin, Higgs Bosons in the Two-Doublet Model with CP Violation, Phys. Rev., 2005, D71, P. 075008. (hep-ph / 0405264 )

M.N. Dubinin, A.V. Semenov, Triple and quartic interactions of Higgs bosons in the two Higgs doublet model with CP violation, Eur. Phys. J. C, 2003, 28, P. 223. (hep-ph / 9812246 )

[5] R. Peccei, H. Quinn, CP Conservation in the Presence of Instantons, Phys.Rev.Lett., 1977, 38, P.1440

[6] A. Brignole, J.R. Espinosa, M. Quiros, F. Zwirner, Aspects of the electroweak phase transition in the minimal supersymmetric standard model, Phys. Lett., 1994, B324 P.181 (hep-ph/9312296)

[7] M. Carena, G. Nardini, M. Quiros, C.E.M. Wagner. The Baryogenesis Window in the MSSM, Nucl.Phys., 2009, B812, P.243 (arXiv:0809.3760 [hep-ph])

M. Carena, G. Nardini, M. Quiros and C.E.M. Wagner, The Effective Theory of the Light Stop Scenario, JHEP, 2008, 10, P.062 (arXiv:0806.4297 [hep-ph])

M. Carena, M. Quiros, C.E.M. Wagner, Opening the window for electroweak baryogenesis, Phys.Lett., 1996, B380, P.81 (hep-ph/9603420)

[8] A.I. Bochkarev, M.E.Shaposhnikov, Electroweak Production of Baryon Asymmetry and Upper Bounds on the Higgs and Top Masses, Mod.Phys.Lett., 1987, A2, P.417

M.E. Shaposhnikov, Possible Appearance of the Baryon Asymmetry of the Universe in an Electroweak Theory, JETP Lett., 1986, 44, P.465 (Pisma Zh.Eksp.Teor.Fiz., 1986, 44, P.364

[9] M. Carena et al., CP violating MSSM Higgs bosons in the light of LEP-2, Phys. Lett., 2000, B495, P. 155. [hep-ph/0009212]

[10] M.Dolgopolov, M.Dubinin, E.Rykova, Critical parameters of the temperature evolution of the two-doublet potential in the minimal supersymmetric standard model, Phys.Atom.Nucl., 2010, 73, P.1032 (Yad.Fiz., 2010, 73, P.1069) 